\title{
Fast closed-loop optimal control of ultracold atoms in an optical lattice
}

\author{
S. Rosi, ${ }^{1}$ A. Bernard, ${ }^{1}$ N. Fabbri, ${ }^{1}$ L. Fallani, ${ }^{1,2}$ C. Fort, ${ }^{1}$ M. Inguscio, ${ }^{1,2}$ T. Calarco, ${ }^{3}$ and S. Montangero ${ }^{3}$ \\ ${ }^{1}$ LENS and Dipartimento di Fisica e Astronomia, Università di Firenze and INO-CNR, 50019 Sesto Fiorentino, Italy \\ ${ }^{2}$ QSTAR Center for Quantum Science and Technology, Largo Enrico Fermi 2, I-50125 Arcetri, Italy \\ ${ }^{3}$ Institut für Quanteninformationsverarbeitung, Universität Ulm, D-89069 Ulm, Germany
}

(Received 25 March 2013; published 1 August 2013)

\begin{abstract}
We present experimental evidence of the successful closed-loop optimization of the dynamics of cold atoms in an optical lattice. We optimize the loading of an ultracold atomic gas minimizing the excitations in an array of one-dimensional (1D) tubes (3D-1D crossover) and we perform an optimal crossing of the quantum phase-transition from a superfluid to a Mott insulator in a 3D lattice. In both cases we enhance the experiment performances with respect to those obtained via adiabatic dynamics, effectively speeding up the process by more than a factor three while improving the quality of the desired transformation.
\end{abstract}

DOI: 10.1103/PhysRevA.88.021601

PACS number(s): 67.85.Hj, 03.67.-a, 05.30.Rt

In the last decade, the implementation of quantum simulators with cold atoms has experienced remarkable expansion [1]. The latest developments in the field have made it possible now to experimentally investigate Fermi and Bose ultracold gases in many different setups [2]. Optical potentials have given access to the simulation of the ground-state physics and the dynamics of some of the most important lattice models: Hubbard and spin models have been successfully implemented [3-7]. Including artificial disorder enables the study of ubiquitous phenomena like Anderson localization [8]. Recently, improved experimental techniques allowed for the acquisition of unprecedented single-atom resolved images and the coherent control of single spins $[9,10]$, paving the way for the next generation of experiments. Novel and more challenging ideas have been proposed to exploit the potential of quantum simulators to study artificial gauge fields related to quantum Hall physics [11], the physics of complex quantum systems [12], and gauge theories [13]. The path towards new experiments of increasing complexity is conditional on the development of better and more precise experimental techniques to achieve increased control on the system under investigation. The necessary steps to be taken are mostly related to technological and experimental development; however, recently important theoretical contributions have been put forward. On one side, shortcuts to adiabaticity strategies might be exploited when analytical insight on the system dynamics are available [14]. On the other side, it has been shown that it is possible to exploit quantum optimal control to synthesize optimal strategies for correlated quantum many-body dynamics [15,16], as already known for fewbody or uncorrelated quantum systems [17-20]. Combining numerical simulations and novel approaches has enabled optimal control of correlated quantum many-body dynamics and optimal driving of phase transitions [15,21] and the engineering of many-body entangled and squeezed states [22]. Despite these promising theoretical results, their experimental implementation might be limited by different issues mainly arising from discrepancies between theoretical models and experimental realization. Even though optimal control fields are generically robust against noise and imperfections [23], it would be desirable to have an optimal control field obtained by means of the most accurate and comprehensive description possible of the system dynamics under consideration. Moreover, there are also cases where open-loop quantum optimal control cannot be applied to a given dynamics as no efficient classical description is available, for example, in highly entangled quantum many-body systems in dimensions greater than one [24]. These limitations might be overcome by means of closed-loop optimal control, i.e., the application of optimal control in a loop that includes experiments, where the control fields are updated at each step after a direct measurement of a figure of merit (FOM) (see Fig. 1 for the case discussed in this Rapid Communication). This approach has the great advantage of taking all sources of uncertainties automatically into account, such as, for example, limited knowledge of system parameters, errors, and constraints. Indeed, closedloop optimization has been successfully applied in different

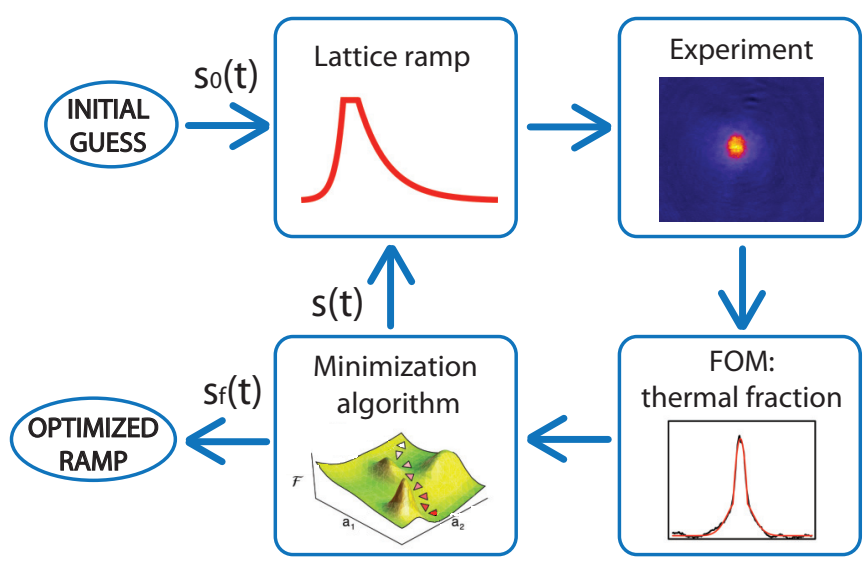

FIG. 1. (Color online) Scheme of closed-loop optimization experiment. The control-field $s(t)$ describes the temporal dependence of the lattice depth during the loading of the atomic gas in the lattice. An initial guess $s_{0}(t)$ is chosen: For each experimental run a time-of-flight image is recorded. From a double-structure fit of the density distribution we extract the figure of merit $F$. The optimization algorithm provides an updated function $s(t)$ and the experiment is repeated until reaching an optimal field $s_{f}(t)$. 
contexts, from NMR to femtosecond laser driving of molecular dynamics [17]. Our approach, based on chopped random basis (CRAB) optimization, has the additional nice feature of avoiding complex broadband control fields, and it is simple to be implemented in the laboratory. On top of that, CRAB optimization might be used to find simple but unknown optimal control fields that might be exploited in similar or following repetitions of the experiment. Indeed, this has been shown to be the case for the open-loop control of Rydberg atom dynamics where a CRAB optimization on different disordered samples guided the design of a robust optimal control field [25].

In this Rapid Communication we apply optimal control theory to give an experimental demonstration of a closed-loop optimal loading of an ultracold atomic sample in optical lattices. We demonstrate the possibility of guiding the system from an initial to a final state through a nonadiabatic transformation, such that the final state is reached in a shorter time and with a better fidelity with respect to a slower quasiadiabatic procedure. We optimize two different fundamental processes that appear in many different cold-atom experiments. We first optimally load a Bose-Einstein condensate (BEC) of ${ }^{87} \mathrm{Rb}$ atoms in a two-dimensional (2D) optical lattice in order to produce an array of 1D gases [26]. Afterwards, we optimally drive an atomic gas across the quantum phase transition from a superfluid to a Mott insulating phase loading the BEC into a 3D optical lattice.

Experimental setup. A degenerate sample of ${ }^{87} \mathrm{Rb}$ is obtained in a hybrid magneto-optical trap realized with the superposition of a quadrupolar magnetic field and a focused red-detuned laser beam [27]. After evaporative cooling, we obtain a BEC of about $2 \times 10^{5}$ atoms experiencing an external harmonic potential with cylindrical symmetry [28]. In the slow adiabatic loading procedure, the lattice potential depth $s(t)$ (expressed in recoil energy units $E_{r}=h^{2} / 2 m \lambda^{2}$, where the optical lattice wavelength is $\lambda=830 \mathrm{~nm}, h$ is the Planck constant, and $m$ is the atomic mass) is increased exponentially from zero to a maximum value $s_{\max }$ in a time $\Delta t$ and a time constant $\tau$ according to

$$
s(t)=s_{\max } \frac{1-e^{t / \tau}}{1-e^{\Delta t / \tau}} .
$$

The lattice intensity is controlled by acousto-optic modulators and stabilized at the desired value by a feedback system during the whole transformation [29]. In the optimization tests, we compare the nonadiabatic transformations to an exponential quasiadiabatic ramp of total duration of $\Delta t_{\mathrm{ad}}=140 \mathrm{~ms}$ and time constant $\tau_{\mathrm{ad}}=30 \mathrm{~ms}$. The latter is representative of typical ramps used in experiments [30]. The estimation of the excitations produced in the gas by the nonadiabatic ramp in obtained in the following way. Once the optical lattice has reached the maximum intensity $s_{\max }$, it is kept at this value for $5 \mathrm{~ms}$ and then turned off with the time-reversed exponential ramp. Then, after a thermalization time $\Delta t_{\text {th }}$, all the other confining potentials are switched off and the atomic cloud evolves in free space. The energy excess of the lattice gas is quantified by measuring the thermal fraction from absorption imaging after a time of flight (TOF) of $28 \mathrm{~ms}$, when the thermal and condensed parts are well distinguishable [31]. The thermal fraction is taken as the FOM to be minimized by the optimization process. Note that the ramping down of the lattice is always adiabatic (exponential decreasing ramp with $\tau_{\mathrm{ad}}$ and $\Delta t_{\mathrm{ad}}$ ), so that the heating measured at the end of this ramp reflects the heating of the gas in the loading procedure.

Optimization. A typical quantum optimal control problem is stated as follows. Given a system described by a Hamiltonian function of a time-dependent control field $s(t)$, i.e., $H=H(s(t))$, we aim to extremize a given FOM $F(s(t))$ after the time evolution driven by $s(t)$ in the time interval $t \in[0, \Delta t]$. CRAB optimization is a strategy that solves the aforementioned problem starting with an initial guess $s_{0}(t)$ and looks for the optimal control field that extremizes the FOM over all the functions of the form $s(t)=s_{0}(t) g(t)$, where the correction $g(t)$ is defined as a truncated expansion in some given basis functions. In particular, here we assume

$$
g(t)=\frac{1+\sum_{j=1}^{n_{f}}\left[a_{j} \sin \left(2 \pi v_{j} t\right)+b_{j} \cos \left(2 \pi v_{j} t\right)\right]}{1+\sum_{j=1}^{n_{f}}\left[a_{j} \sin \left(2 \pi v_{j} \Delta t\right)+b_{j} \cos \left(2 \pi v_{j} \Delta t\right)\right]}
$$

for some (small) number $n_{f}$ of components with frequencies $v_{j}$. The optimization problem is then recast as an extremization of a multivariable function $F\left(\left\{h_{j}\right\}\right)$ of the variables $h_{j}=$ $\left(a_{j}, b_{j}\right)$. Here the minimization is performed by means of a simplex minimization algorithm [15].

In optical lattice experiments, this problem typically appears as one aims to prepare a given state varying the optical lattice intensity $s(t)$. Indeed, our goal is to prepare the system ground state, thus minimizing the residual excitations in the final state. In particular, first we optimize the loading of a BEC in a 2D optical lattice as a test of our closed-loop experiment. Then we exploit the CRAB method to optimize the superfluid-Mott insulator quantum phase transition. The experimental procedure of closed-loop optimization is shown in Fig. 1: We define an initial guess $s_{0}(t)$ and implement the experimental sequence described above, at the end of which a TOF image is recorded. An automated fitting procedure results in a measurement of the final thermal fraction of the sample $\mathrm{TF}=N_{\text {th }} / N_{\text {tot }}$ to be compared with the initial thermal fraction $\mathrm{TF}_{i}$ (where $N_{\text {th }}$ is the number of atoms in the thermal component and $N_{\text {tot }}$ is the total atom number of the sample). Their ratio defines the FOM $F=\mathrm{TF} / \mathrm{TF}_{i}$ we minimize. With this information the minimization subroutine implements a search in the parameters space that defines an updated loading ramp $s(t)$. The loop is then closed and the process repeated until convergence or when the given desired precision has been reached.

$3 D-1 D$ crossover. We first consider the transformation between a 3D BEC and an array of 1D quasicondensates obtained loading the BEC in a 2D lattice. As a warmup for the full CRAB optimization, here we optimize the process over a restricted class of functions, namely loading ramps of exponential shape with different duration $\Delta t$ and time constant $\tau$ as defined in Eq. (1). The final value of the two lattices' intensity $s_{\max }=32$ is high enough to produce an array of independent $1 \mathrm{D}$ gases where the transverse degrees of freedom are completely frozen and the tunneling rate of atoms between different sites is negligible on the time scale of the experiment. The thermalization time is $\Delta t_{\text {th }}=1 \mathrm{~s}$. Before running the optimization algorithm, as the final thermal fraction is a $2 \mathrm{D}$ function of the free parameters $(\Delta t, \tau)$ and thus 

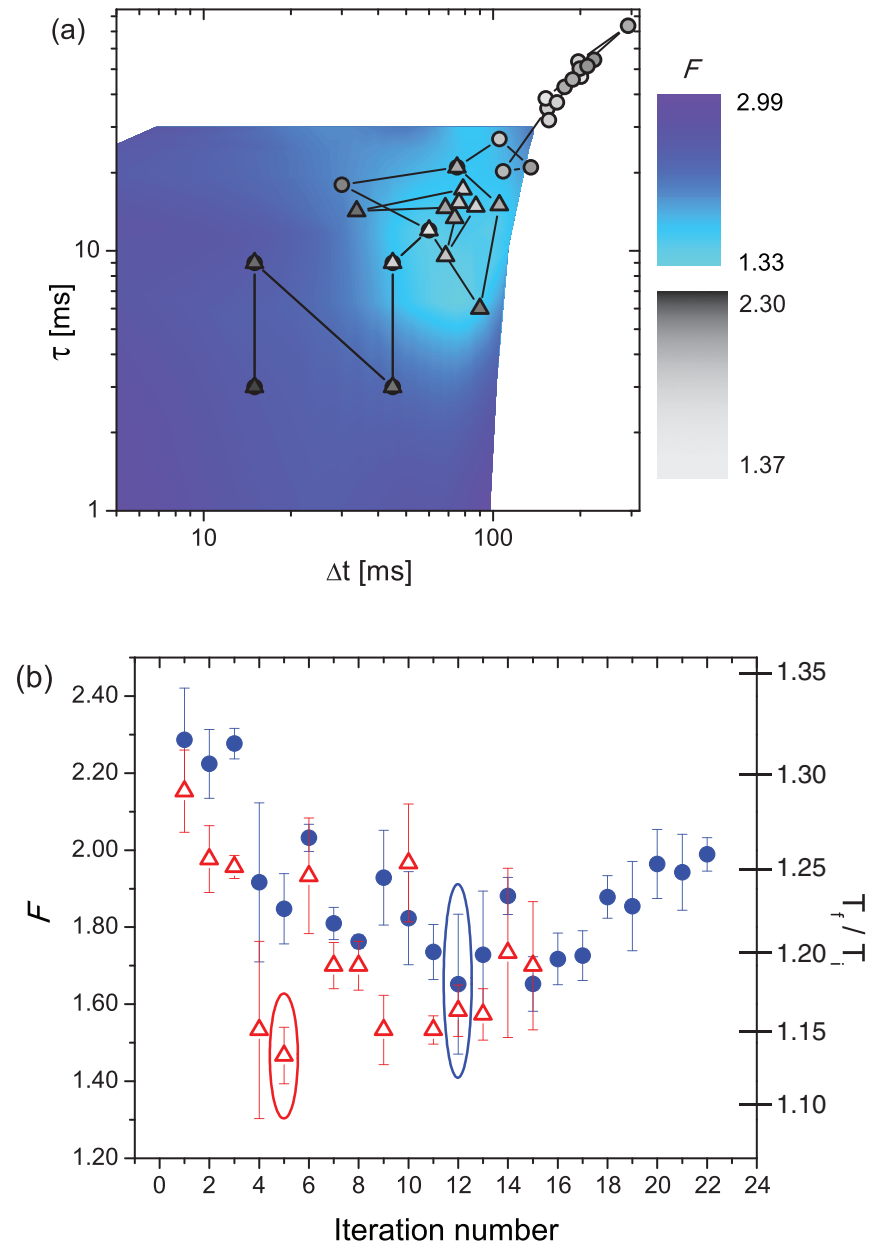

\begin{tabular}{|c|c|c|c|}
\hline & $\Delta t(\mathrm{~ms})$ & $\tau(\mathrm{ms})$ & $F$ \\
\hline quasi-ad. & 140 & 30 & $1.66 \pm 0.02$ \\
$s_{0}$ & 15 & 3 & $2.30 \pm 0.03$ \\
$s_{\text {opt }}$ RUN 1 & 154 & 35 & $1.73 \pm 0.02$ \\
$s_{\text {opt }}$ RUN 2 & 45 & 9 & $1.40 \pm 0.06$ \\
\hline
\end{tabular}

FIG. 2. (Color online) 3D-1D crossover. (a) Two-dimensional mapping (blue-colored palette) of the FOM as a function of the parameters $\Delta t$ and $\tau$. Circles (triangles) show the progress during the first (second) run of optimization (gray-colored palette). (b) Measured figure of merit $F$ (ratio between final and initial thermal fraction of the atomic sample) after ramping up and down a 2D optical lattice during the optimization loop. The first (second) experimental run (see text) is represented by blue circles (red triangles). The two best results $F_{\mathrm{opt}}$ measured in the two runs are circled in evidence. For completeness, on the right side of the graph we report also the corresponding values of the ratio between temperature $T_{f}$ measured after having switched on and off the lattices and temperature $T_{i}$ measured before loading the lattices (inferred from the thermal fraction assuming thermal equilibrium). The table reports the values of the FOM corresponding respectively to the quasiadiabatic loading, the exponential initial loading $\left(s_{0}\right)$ and the best one $\left(s_{\text {opt }}\right)$ of the two runs, together with the correspondent values of the parameters $\Delta t$ and $\tau$.

easily representable, we plotted it in the $2 \mathrm{D}$ parameter phase space [colored map in Fig. 2(a)]. This allows the results of the closed-loop optimization to be compared with a brute-force approach, that is, an extensive search in the parameter space.
The colored map gives us interesting information on the problem structure; notice, for example, that for very short ramps the FOM is clearly higher than in cases of longer ones, as expected. The extensive mapping approach is unfeasible as soon as the number of the parameters increases; however, in this case where only two parameters are considered we show that the optimization finds very quickly the minima. Figure 2 presents two tests of the optimization loop characterized by the same initial guesses $\Delta t_{0}=15 \mathrm{~ms}$ and $\tau_{0}=3 \mathrm{~ms}: F$ is reported for both runs in Fig. 2(b) as a function of the iteration number $n$ of the optimization loop. The possibility of finding different final results is due to the fact that performing an experiment characterized by a finite number of iterations and experimental errors, we may bump into little deviations in the measurements from run to run. As can be seen, in both cases after a quick convergence to a minimum the algorithm looks for other solutions possibly present in case the first was a local minimum, but it found none. In Fig. 2(a) the trajectories in the space of parameters clearly reflect this behavior. Finally, as reported in the table in Fig. 2, the two loops give two pairs of different final values $\left(\Delta t_{\mathrm{opt}}, \tau_{\mathrm{opt}}\right)$ : The second run ended in an improved result, while the first missed the global minima, spending the available iterations in the large region where the FOM is almost flat, as we can see in Fig. 2(a).

Superfluid-Mott insulator transition. We now apply the closed-loop optimization to a more complex dynamical process, that is, the loading of the BEC into a 3D lattice, effectively optimally driving the quantum phase transition from a superfluid to a Mott insulator phase. We perform a full CRAB optimization; that is, we search for the best possible correction of the form introduced in Eq. (2) to an initial exponential ramp $s_{0}(t)$ given in Eq. (1). Here we use two frequency components; i.e., we perform the optimization in a $4 \mathrm{D}$ parameter space, with $v_{1}=1 / \Delta t$ and $v_{2}=2 / \Delta t$, where $\Delta t=40 \mathrm{~ms}$ and $\tau=8 \mathrm{~ms}$. The final optical lattice depth is $s_{\max }=25$-deeply in the Mott insulating phase-and the thermalization time $\Delta t_{\mathrm{th}}=200 \mathrm{~ms}$. As in the previous case, the FOM is represented by the normalized thermal
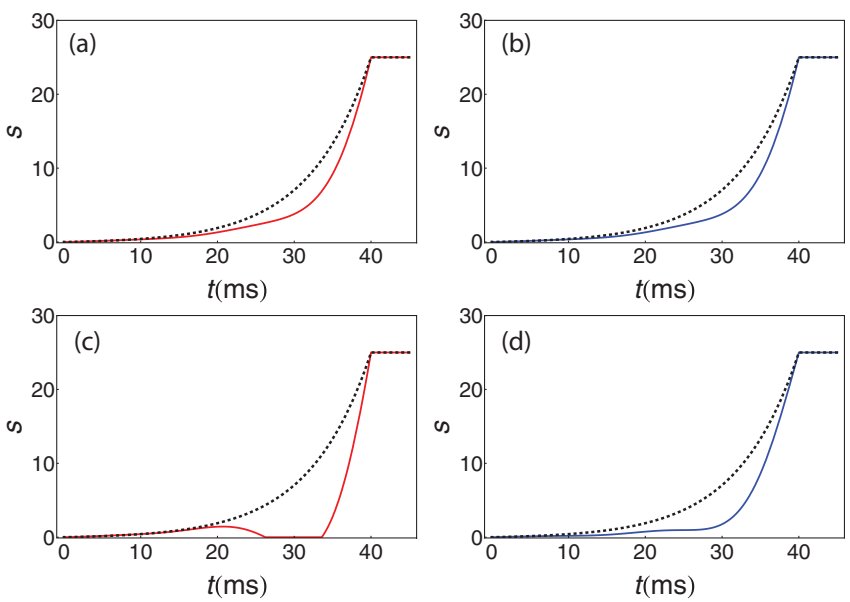

FIG. 3. (Color online) Optical lattice depth ramps (in $E_{r}$ units) $s(t)$ for the superfluid-Mott insulator transition. Solid lines represent (a) first-run initial guess, (b) first-run optimized ramp, (c) second-run initial guess, and (d) second-run optimized ramp. The dashed black line represents the exponential noncorrected ramp. 


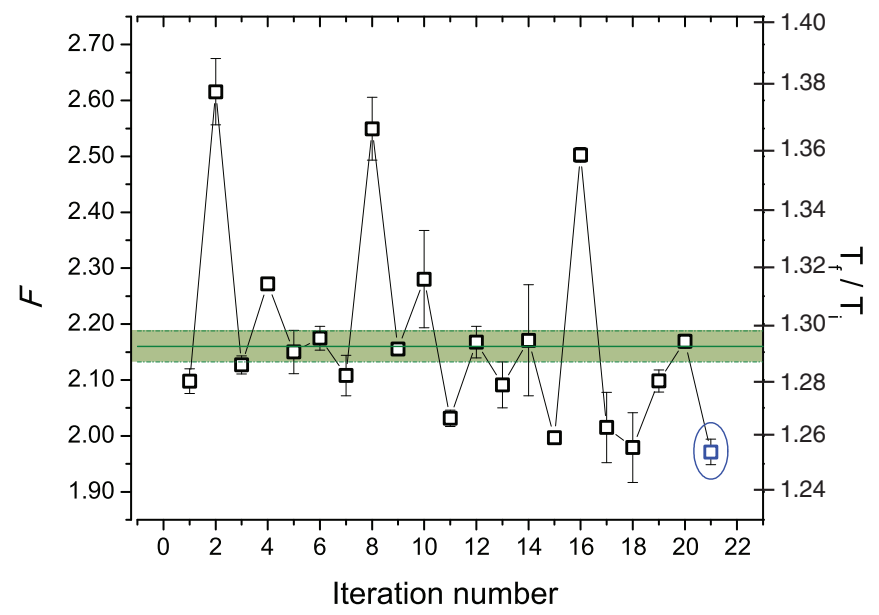

\begin{tabular}{|c|c|c|c|c|}
\hline & $\begin{array}{c}\tau \\
(\mathrm{ms})\end{array}$ & $\begin{array}{c}\Delta t \\
(\mathrm{~ms})\end{array}$ & {$\left[a_{1}, b_{1}, a_{2}, b_{2}\right]$} & $F$ \\
\hline quasi-ad. & 140 & 30 & {$[0,0,0,0]$} & $2.16 \pm 0.03$ \\
$s_{u c}$ & 40 & 8 & {$[0,0,0,0]$} & $2.19 \pm 0.03$ \\
$s_{\text {opt }}$ RUN 1 & 40 & 8 & {$[0.2,0.2,0.1,0.1]$} & $1.89 \pm 0.03$ \\
$s_{\text {opt }}$ RUN 2 & 40 & 8 & {$[-0.09,-0.22,0.70,0.13]$} & $1.97 \pm 0.02$ \\
\hline
\end{tabular}

FIG. 4. (Color online) Optimization of the superfluid-Mott insulator transition. The FOM $F$ is reported as a function of the iteration number $n$ (second run). The green region shows the FOM $F_{\text {ad }}=(2.16 \pm 0.03)$ in the case of the quasiadiabatic loading ( $\Delta t=140 \mathrm{~ms}, \tau=30 \mathrm{~ms}$ ). The blue-circled empty square reports the final optimal loading $F_{\text {opt }}$. In the table are reported the values of the parameters $\left[a_{1}, b_{1}, a_{2}, b_{2}\right]$ of the correction and the FOM $F$ corresponding, respectively, to the quasiadiabatic loading, to the exponential uncorrected loading $\left(s_{u c}\right)$, and to the optimal loading $\left(s_{\text {opt }}\right)$ found in the two runs.

fraction $F$, observed after an adiabatic switching off of the lattices, which measures the residual excitations on top of the Mott ground state. In this case, the quasiadiabatic ramp $\left(\Delta t=140 \mathrm{~ms}, \tau=30 \mathrm{~ms}\right.$ ) gives $F_{\text {ad }} \sim 2.16$ (green line in Fig. 4). We have performed two different optimization runs characterized by different sets of initial random parameters $\left\{h_{j}\right\}$. In the first one the initial ramp is very close to the guess $s_{0}(t)$, while in the latter it is characterized by a very strong correction. The resulting optimal ramps are shown in Fig. 3, together with the initial ramp and the uncorrected (pure exponential) one. It may be interesting to note that, despite starting from very different initial guesses, the two loops result in two very similar output ramps, characterized by a slow variation in the first part and a fast increase in the second part. The trend of $F$ during the second run is shown in Fig 4. The results of the two optimizations are summarized in the table. Note that, although the number of parameters involved in the optimization is very small $\left(n_{f}=2\right)$, we obtain a transformation about three times faster than the adiabatic one and with a final temperature improved by a few percent. Whenever additional improvements would be needed for real applications, where improved precisions and/or faster ramps are crucial elements for a successful experiment-for example, to reduce decoherence effects - the presented optimization can be improved, adding more optimization parameters and/or increasing the optimization runs.

Conclusions. We have shown that closed-loop optimal control can be effectively applied to the manipulation of cold atoms in optical lattices, demonstrating how it is possible to develop strategies different from adiabatic ones. In particular, we have shown that it is possible to drive the 3D-1D crossover and the 3D superfluid-Mott insulator transition over time scales about one-third shorter than a standard quasiadiabatic one, while still improving on the final state. Our strategy can be applied to the implementation of the ground state of different Hamiltonians. This demonstration paves the way to further developments that will make it possible to face the challenges of the next-generation experiments with cold atoms in optical lattice.

Acknowledgments. We acknowledge support from EU IP Project AQUTE and Project EU-STREP MALICIA. The work at LENS has been supported by MIUR through Grant No. PRIN2009, ERC Advanced Grant No. DISQUA, IIT Seed Project ENCORE. T.C. and S.M. acknowledge support from the DFG via Grant No. SFB/TRR21.
[1] I. Bloch, J. Dalibard, and S. Nascimbène, Nat. Phys. 8, 267 (2012).

[2] I. Bloch, J. Dalibard, and W. Zwerger, Rev. Mod. Phys. 80, 885 (2008).

[3] D. Greif, T. Uehlinger, G. Jotzu, L. Tarruell, and T. Esslinger, Science 340, 1307 (2013).

[4] M. Greiner, O. Mandel, T. Esslinger, T. W. Hänsch, and I. Bloch, Nature (London) 415, 39 (2002).

[5] R. Jördens, N. Strohmaier, K. Günter, H. Moritz, and T. Esslinger, Nature (London) 455, 204 (2008).

[6] U. Schneider, L. Hackermüller, S. Will, Th. Best, I. Bloch, T. A. Costi, R. W. Helmes, D. Rasch, and A. Rosch, Science 322, 1520 (2008).

[7] J. Simon, W. S. Bakr, R. Ma, M. E. Tai, P. M. Preiss, and M. Greiner, Nature (London) 472, 307 (2011).
[8] J. Billy, V. Josse, Z. Zuo, A. Bernard, B. Hambrecht, P. Lugan, D. Clément, L. Sanchez-Palencia, P. Bouyer, and A. Aspect, Nature (London) 453, 891 (2008); G. Roati, C. D'Errico, L. Fallani, M. Fattori, C. Fort, M. Zaccanti, G. Modugno, M. Modugno, and M. Inguscio, Nature (London) 453, 895 (2008).

[9] J. F. Sherson et al., Nature (London) 467, 68 (2010).

[10] C. Weitenberg et al., Nature (London) 471, 319 (2011).

[11] F. Gerbier and J. Dalibard, New J. Phys. 12, 033007 (2010).

[12] S. Montangero, D. Frustaglia, T. Calarco, and R. Fazio, Europhys. Lett. 88, 30006 (2009).

[13] D. Banerjee, M. Dalmonte, M. Müller, E. Rico, P. Stebler, U.-J. Wiese, and P. Zoller, Phys. Rev. Lett. 109, 175302 (2012). 
[14] J. G. Muga, X. Chen, E. Torrontegui, S. Ibez, I. Lizuain, A. Ruschhaupt, J. Phys.: Conf. Ser. 306, 012022 (2011).

[15] P. Doria, T. Calarco, and S. Montangero, Phys. Rev. Lett. 106, 190501 (2011).

[16] T. Caneva, T. Calarco, and S. Montangero, Phys. Rev. A 84, 022326 (2011).

[17] D. J. Tannor and S. A. Rice, J. Chem. Phys. 83, 5013 (1985); P. Brumer and M. Shapiro, Annu. Rev. Phys. Chem. 43, 257 (1992); M. Dahleh, A. Peirce, H. Rabitz, and V. Ramakrishna, Proc. IEEE 84, 7 (1996); L. Viola and S. Lloyd, Phys. Rev. A 58, 2733 (1998); T. C. Weinacht, J. Ahn, and P. H. Bucksbaum, Nature (London) 397, 233 (1999); C. Brif, R. Chakrabarti, and H. Rabitz, New J. Phys. 12, 075008 (2010).

[18] A. Rahmani, T. Kitagawa, E. Demler, and C. Chamon, Phys. Rev. A 87, 043607 (2013).

[19] S. Machnes, M. B. Plenio, B. Reznik, A. M. Steane, and A. Retzker, Phys. Rev. Lett. 104, 183001 (2010).

[20] R. Bücker, J. Grond, S. Manz, T. Berrada, T. Betz, C. Koller, U. Hohenester, T. Schumm, A. Perrin, and J. Schmiedmayer, Nat. Phys. 7, 608 (2011).

[21] T. Caneva, T. Calarco, R. Fazio, G. E. Santoro, and S. Montangero, Phys. Rev. A 84, 012312 (2011).

[22] T. Caneva, T. Calarco, and S. Montangero, New J. Phys. 14, 093041 (2012); T. Caneva, S. Montangero, M. Lukin, and T. Calarco, arXiv:1304.7195.

[23] S. Montangero, T. Calarco, and R. Fazio, Phys. Rev. Lett. 99, 170501 (2007); T. Caneva, A. Silva, R. Fazio, T. Calarco, and S. Montangero, arXiv:1301.6015.
[24] G. Vidal, Phys. Rev. Lett. 93, 040502 (2004); G. Evenbly and G. Vidal, arXiv:1210.1895.

[25] M. M. Müller, A. Kölle, R. Löw, T. Pfau, T. Calarco, and S. Montangero, Phys. Rev. A 87, 053412 (2013).

[26] T. Kinoshita, T. Wenger, and D. S. Weiss, Science 305, 1125 (2004); B. Paredes, A. Widera, V. Murg, O. Mandel, S. Fölling, I. Cirac, G. V. Shlyapnikov, T. W. Hänsch, and I. Bloch, Nature (London) 429, 277 (2004); B. Laburthe Tolra, K. M. O'Hara, J. H. Huckans, W. D. Phillips, S. L. Rolston, and J. V. Porto, Phys. Rev. Lett. 92, 190401 (2004); E. Haller, M. Gustavsson, M. J. Mark, J. G. Danzl, R. Hart, G. Pupillo, and H.-C. Nägerl, Science 325, 1224 (2009).

[27] Y.-J. Lin, A. R. Perry, R. L. Compton, I. B. Spielman, and J. V. Porto, Phys. Rev. A 79, 063631 (2009).

[28] The characteristic frequencies of the harmonic trapping experienced by atoms are about $(40 \times 9 \times 40 \mathrm{~Hz})$.

[29] The bandwidth of our stabilization loop, in the $\mathrm{kHz}$ range, allows the accurate implementation of the lattice ramps and the cancellation of most of the mechanical noise. Any residual noise contribution as well as any other experimental imperfection is intrinsically included in the learning closed-loop, which comes out with the best result tailored for the specific experimental conditions.

[30] T. Gericke, F. Gerbier, A. Widera, S. Fölling, O. Mandel, and I. Bloch, J. Mod. Opt. 54, 735 (2007).

[31] Note that the measurement is performed after the lattice switching off, since we do not have access to direct information on temperature and/or excitations. 